\title{
Über die lonensorption und die Protolysengleichgewichte von Holz in Elektrolyten
}

\section{Journal Article}

Author(s):

Popper, Rudolf

Publication date:

1978

Permanent link:

https://doi.org/10.3929/ethz-b-000422732

Rights / license:

In Copyright - Non-Commercial Use Permitted

Originally published in:

Holzforschung 32(3), https://doi.org/10.1515/hfsg.1978.32.3.77 


\title{
Über die Ionensorption und die Protolysengleichgewichte
} von Holz in Elektrolyten

\author{
Von Rudolf Popper \\ Institut für Mikrotechnologische Holzforschung, ETH Zürich
}

Schlüsselwörter (Sachgebiete)

Adsorption

Elektrolyte

Ionenaustausch

Austausch-Kapazität

Ionisierungskonstanten

Sorptionsmechanismus

Tannenholz

(Abies alba)

\author{
Überdie Ionensorption und die Protolysengleichgewichte von Holz in Elektrolyten \\ Zusammenfassung
}

Aufgrund der vorgenommenen Untersuchungen zeigt das Holz Eigenschaften eines bifunktionellen Polyelektrolyts, wobei im untersuchten pH-Bereich ein Kationenaustausch bewiesen werden konnte. Während der Protolyse von Tannenholz machen sich zwei ionenaktive Gruppen bemerkbar. Die Ionenaktivität dieser ladungstragenden Gruppen innerhalb des Protolysengleichgewichtes wurde für die Bestimmung der Ionenaustausch-Kapazität genutzt. Sie wurde durch potentiometrische Titration ermittelt und beträgt $0,016 \pm 0,007$ Milliäquivalente bezogen auf das Trockengewicht des Holzes. Die Ionenstärke-Abhängigkeit der Gegenionen-Aufnahme sowie die Stoffbilanz entlang der Protolyse zeigen, daß der Ionenaustausch nicht der einzige Sorptionsmechanismus darstellt. Um die Bindungsstellen am Holz konkurrieren die Gegenionen mit den Ionen des Lösungsmittels. Das Holz nimmt nicht nur die Gegenionen bzw. die Ionen des Lösungsmittels, sondern auch die zu den Gegenionen gehörenden, jedoch entgegengesetzt geladenen Ionen auf. Außer der Ionen-Bindung sind bei der Sorbat-Aufnahme auch van der Waals'sche und Kapillarkräfte im Spiel.

Für die Schätzung der Gleichgewichtskonstanten von beiden im Holz befindlichen ionogenen Gruppen wurde die Bestimmung der Gegenionen-Konzentration vorgenommen. Die Analyse der Gegenionen-Konzentration als Funktion des pH-Wertes ergab Aciditätskonstanten pKć $=5,8$ $\pm 0,9$ und $\mathrm{pK}_{\mathrm{c}}^{\prime \prime}=10,4 \pm 0,4$. Diese entsprechen mit größter Wahrscheinlichkeit einerseits den Carboxyl- und anderseits den phenolischen Hydroxylgruppen des Holzes.

Keywords

Adsorption

Electrolytes

Ion-exchange

Exchange capacity

Ionization constants

Sorption mechanism

Silver fir

\section{Ion-Absorption and Protolytic Equilibrium of Wood in Electrolytes}

\section{Summary}

According to the experiments wood shows bi-functional polyelectrolytic properties. Evidence has been adducted that wood shows in a designated pH-range cation exchange capacity. In the protolytic reaction of fir wood (Abies alba Mill.) two surface active groups of ions are present. The ion activity of these charged groups within the protolytic equilibrium was used to determine ionexchange capacity. It was determined by potentiometric titration and measured $0.016 \pm 0.007$ milliequivalents based on dry weight of wood. The ionic strength dependency of the opposite ion regain and the electrolyte regain (indicated by mass balance) shows that ion exchange is not the only sorption mechanism in function during the protolytic reaction. The opposite ions together with the ions of the dissolving agent, compete for a place in the binding position. The wood absorbs not only the opposite ions (along with the ions of the dissolving agent), but also ions of opposite polarity to them. In addition to the electrostatic bond of the sorbate-regain, Van der Waals and capillary forces are involved.

The concentration of opposite ions are previously determined in order to estimate the equilibrium constants of the two ionic groups in the wood. The analysis of the opposite ion concentration as a function of the $\mathrm{pH}$ value gave acidity constants of $\mathrm{pK} \mathrm{c}^{\prime}=5.8 \pm 0.9$ and $\mathrm{pK}_{\mathrm{c}}^{\prime \prime}=10.4 \pm 0.4$. It is highly probable that these represent the carboxyl groups as well as the phenolic hydroxyl groups of the wood.'

\section{r. Einleitung}

Das Holz ist in seiner chemischen Struktur gekennzeichnet durch zahlreiche dissoziationsfähige Molekülgruppen; sie lassen auf einen elektrolytischen Charakter schließen. Diese Eigenschaft ist von einigen Forschern erkannt worden, und es wurde auch auf verschiedene Art versucht, davon Gebrauch zu machen. Bemerkenswert in dieser Hinsicht sind Arbeiten von Kratzl (1955), in denen angestrebt wird, durch Sulfurierung, Sulfitierung und Oxidation das $\mathrm{Holz}$ in einen technisch verwendbaren Kationenaustauscher umzu- wandeln. Auf die Ionenaustauscheigenschaften des Zellstoffes machten Ohlss on (1975) und Rosen (1975) aufmerksam. Aber auch für die Imprägnierung von Holz mit ionogenen Holzschutzmitteln scheint der elektrolytische Charakter des Holzes eine wichtige Rolle zu spielen. Die Aufnahme von anorganischen Stoffen durch das Holz schrieb Bauch (1964) schließlich indirekt dem ionogenen Charakter des Holzes zu, wobei er eine überwiegende Kationen-Aufnahme feststellte.

Da die nähere Kenntnis der elektrolytischen Eigenschaften von Holz für die theoretische und praktische 
Holzforschung von Bedeutung ist, sind Untersuchungen der grundlegenden Ionensorptionseigenschaften des Holzes an unserem Institut aufgenommen worden. Wir sehen deren Bedeutung in Hinsicht auf das technologische Verhalten von Holz: indem die Imprägnierung mit dissoziierten oder dissoziationsfähigen Lösungen anhand der Möglichkeit einer selektiven IonenAufnahme durch Regulierung des $\mathrm{pH}$-Wertes studiert werden kann; sodann ist die Beeinflussung der Quellungs- und Schwindungseigenschaften des Holzes durch $\mathrm{pH}$ - und Ionenstärke-Änderungen zu klären. Aus dem Gebiet der theoretischen Holzforschung ergeben sich ebenfalls Fragen, die aller Voraussicht nach leichter verständlich werden, wenn das elektrolytische Verhalten der Zellwände in Betracht gezogen wird. Im Vordergrund stehen hier Probleme der Transportmechanismen über die Membranen und die Zell-Einheiten hinweg. In den pflanzlichen Organen können Dissoziationsvorgänge ganz allgemein als treibende Kräfte für die Transportphänomene angenommen werden. In der Literatur findet man Anhaltspunkte, die diese Annahme indirekt unterstützen - sei es im Sinne der lokalen $\mathrm{H}^{+}$-Konzentration, die die Permeabilität der Membranen aktiviert, oder die relative Selektivität der Wurzelzellen zu $\mathrm{K}^{+}$-Ionen, die proportional zu der $\mathrm{H}^{+}$Konzentration sein sollte (Pitman 1976). Ebenso könnte versucht werden, die Inkrustierungseffekte von pflanzlichen Membranen (Alterungseffekte) durch Veränderungen innerhalb der Dissoziationsgleichgewichte zu klären (Bosshard 1977).

\section{Theoretische Grundlagen,}

\section{Definition und Problemstellung}

Zerfällt ein Molekül in einer Lösung in geladene Ionen, so wird das Molekül als Elektrolyt und der Vorgang als elektrolytische Dissoziation bezeichnet. Demnach ist die Dissoziationsfähigkeit ein charakteristisches Merkmal eines Elektrolyten. Am Beispiel einer schwachen Säure kann man die Dissoziation folgendermaßen darstellen: Säuren reagieren mit Wasser unter Bildung von Hydroxoniumionen und den entsprechenden Anionen

$$
\mathrm{HA}+\mathrm{H}_{2} \mathrm{O} \rightleftharpoons \mathrm{H}_{3} \mathrm{O}^{+}+\mathrm{A}^{-}
$$

Die Lage des Gleichgewichtes dieser Reaktion wird durch die Dissoziationskonstante beschrieben:

$$
\mathrm{K}=\left(\mathrm{a}_{\mathrm{H}, \mathrm{O}^{+}}\right) \cdot\left(\mathrm{a}_{\mathrm{A}}-\right) /\left(\mathrm{a}_{\mathrm{HA}}\right) \text {, }
$$

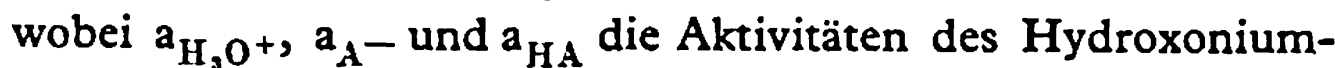
ions, des Anions und des undissoziierten Elektrolyten sind.

Die Änderung der freien Enthalpie ist eine thermodynamische Größe, die die Affinitätsverhältnisse einer Reaktion beschreibt. Die Beziehung zwischen freier Enthalpieänderung $(\Delta \mathrm{G})$ und der Dissoziationskonstante $(\mathrm{K})$ ist durch folgende Gleichung gegeben:

$$
\Delta \mathrm{G}=\mathrm{R} \cdot \mathrm{T} \cdot \mathrm{pK},
$$

wobei $R$ die Gaskonstante, $T$ die absolute Temperatur und $p K$ der negative Logarithmus der Dissoziationskonstanten sind.

So kann man die Säure-Basen Gleichgewichte über die Dissoziationskonstante als ein Maß der Reaktivität eines Elektrolyten betrachten.

Der elektrolytische Charakter eines organischen Stoffes ist durch seine Struktur geprägt. Besitzt ein Elektrolyt wenigstens ein makromolekulares Ion, wird er als Polyelektrolyt bezeichnet. Elektrochemische Eigenschaften der Polyelektrolyten sind durch den polymeren Charakter weitgehend beeinflußt. Beim Verdünnen der niedermolekularen Elektrolyten werden die geladenen Teilchen voneinander getrennt, so daß sich diese nicht gegenseitig beeinflussen können. Im Gegensatz dazu wird bei den Polyelektrolyten sogar bei extremer Verdünnung eine relativ hohe Ladungsdichte beibehalten. Im Unterschied zum niedermolekularen Elektrolyten ist das Dissoziationsgleich- gewicht des Polyelektrolyten von der Molekularstruktur abhängig.

Die Beziehung zwischen dem pH-Wert und pK-Wert kann man aus der Gleichung $\mathrm{I}$ ableiten indem man das Verhältnis $\left(a_{A^{-}}\right) /\left(a_{H_{A}}\right)$ durch den Dissoziationsgrad $\alpha$ ausdrückt:

$$
\left(\mathrm{a}_{\mathrm{A}^{-}}\right) /\left(\mathrm{a}_{\mathrm{HA}}\right)=\alpha /(\mathrm{I}-\alpha) \text {, }
$$

Durch Einsetzen der Gleichung 4 in die Gleichung 2 ergibt sich nach dem Logarithmieren die Hendersson-Hasselbach'sche Beziehung:

$$
\overline{\mathrm{pH}}=\mathrm{pK}-\log (\mathrm{I}-\alpha / \alpha),
$$

wobei $\overline{\mathrm{pH}}$ der $\mathrm{pH}-$ Wert im Holz bedeutet.

Aus der Gleichung 5 geht hervor, daß der pK-Wert der aktiven Gruppen dem $\overline{\mathrm{pH}}$-Wert des Holzes im Halbneutralisationspunkt $(\alpha=0,5)$ gleichgesetzt werden kann.

Der $\overline{\mathrm{pH}}$-Wert im Holz stimmt mit dem in der Lösung nicht überein. Setzt man voraus, daß das Verhältnis zwischen den Ionen im Flolz und in der Lösung gleich ist:

$$
\left[\overline{\mathrm{H}}^{+}\right]=\left[\mathrm{H}^{+}\right] \cdot\left[\overline{\mathrm{K}}^{+}\right] /\left[\mathrm{K}^{+}\right] \text {, }
$$

wird folglich bei $\alpha=0,5$

$$
\mathrm{pK}_{C}=\overline{\mathrm{pH}}=\mathrm{pH}+\log \left[\mathrm{K}^{+}\right]-\log \left(\left[\overline{\mathrm{X}}^{+}\right] / 2\right),
$$

wobei $\mathrm{pK}_{C}$ der negative Logarithmus der scheinbaren Gleichgewichtskonstante, $\left[\overline{\mathrm{X}}^{+}\right]$die Konzentration der aktiven Gruppen und $\left[\mathrm{K}^{+}\right]$die Gegenion-Konzentration sind (Helfferich 1959).

Beim Eintauchen von Holz in eine wäßrige Lösung eines neutralen Salzes haben wir eine Senkung des pHWertes dieser Lösung feststellen können. Der Grund liegt darin, daß die durch die Dissoziation entstehenden Ionen an der Sorbensoberfläche nicht gleichmäßig sorbiert, sondern einige Ionen bevorzugt werden. Wir sprechen dann von einer Ionen-Sorption und verstehen darunter, daß allgemein ein Ion des Elektrolyten stärker sorbiert wird als das andere. Falls bei der selektiven Adsorption die Ionen des Sorbens und des Elektrolyten ausgetauscht werden, wird sie als eine IonenaustauschSorption und das Sorbens wird als Ionenaustauscher bezeichnet.

Ein Ionenaustauscher besteht aus einer unlöslichen Matrix mit fest gebundenen Ionen sowie aus frei beweglichen Gegenionen, die die Ladung der gebundenen Gruppen kompensieren. Trägt das Sorbens an seinem Gerüst negativ geladene Gruppen mit positiv geladenen Gegenionen, kann es Kationen austauschen und wird deswegen Kationenaustauscher genannt. Entsprechend würde ein Anionenaustauscher angekoppelte positive Gruppen und austauschbare negative Gegenionen enthalten.

Falls das Holz Eigenschaften eines Polyelektrolyten aufweisen sollte, müßte es dissoziierte oder mindestens dissoziationsfähige Molekülgruppen besitzen. Diese Voraussetzung ist anscheinend gegeben, da das Holz ladungstragende alkoholische und phenolische Hydroxylgruppen sowie Carboxylgruppen am hochpolymeren räumlichen Netzwerk trägt. Diese Molekülgruppen sind mittels kovalenter Bindungen an das KohlenstoffGerüst gebunden, wobei innerhalb der Gruppen Ionenbindungen bestehen. Diese Ionen-Bindungen geben Anlaß dazu, daß das Holz als eine Polysäure mit einem polymeren Anion und einem freien beweglichen Gegenion betrachtet werden kann. Unter dieser Annahme dürfte die Dissoziationskonstante des Holzes gemäß Gleichung 2 als Aktivität der Hydroxoniumionen innerhalb der Gleichgewichte bestimmt werden. $\mathrm{Zu}$ diesem Zweck wird das Holz mit gewöhnlichen Basen titriert. Das Holz selbst bleibt dabei praktisch unlöslich, es setzt sich jedoch mit der Reaktionslösung ins Gleichgewicht. Die Protolyse kann durch Messung des pH-Wertes der Lösung während der Titration verfolgt werden und der pK-Wert der aktiven Gruppen aus dem Verlauf der Titrationskurve bestimmt werden. 


\section{Versuchsergebnisse und Diskussion}

Alle Untersuchungen wurden an Probenklötzchen $(2 \times 2$ $\times 2 \mathrm{~cm}$ ) aus Weißtanne (Abies alba Mill.), deren Jahrringe parallel zu den Seiten des Probenkörpers verliefen, angestellt.

\section{I. Bestimmung der nutzbaren Ionen-Sorptionskapazität}

Die Ionen-Sorptionskapazität ist ein quantitatives $M a ß$ für die Fähigkeit eines Sorbens Gegenionen aufzunehmen. Sie hängt von der Zugänglichkeit der funktionellen Gruppen, von der Gegenionen-Konzentration und von der Ionenstärke des Trennmediums ab. Für die Kapazitätsbestimmung wurde die potentiometrische Titration angewendet, die nur die ausnutzbare Kapazität erfaßt. Die Potentialdifferenz wurde mit Hilfe einer kombinierten Meßkette, bestehend aus einer Glas-Indikationselektrode und $\mathrm{Ag} / \mathrm{AgCl}$-Referenzelektrode, mittels eines $\mathrm{pH}$-Meters (Auflösungsvermögen o,or pH) gemessen. Da die Aufnahme der Titrationskurve mit nur einer einzigen Probe viel Zeit in Anspruch nehmen würde, wurde eine Reihe von darrgetrockneten Proben in Schliffkolben eingewogen, mit abgestufter Menge eingestellter $0,02 \mathrm{~N} \mathrm{KOH}$ (Faktorstellung gegen Oxalsäure als Urtiter) und mit deionisiertem Wasser auf $100 \mathrm{ml}$ aufgefüllt, wobei eine Probe ohne Zusatz von $\mathrm{KOH}$ nur mit $100 \mathrm{ml}$ Wasser belassen wurde. Um den Pipettierfehler möglichst tief $\mathrm{zu}$ halten, wurden alle Lösungen mit Kolbenbüretten (Metrohm E 412, Dosiergenauigkeit 0, I \%) abgemessen. Als Kontrollreihe dienten die gleichen Lösungen jedoch ohne Holzproben. Die Kolben wurden dann so lange geschüttelt bis der pH-Wert der Lösungen konstant blieb. Zur Ermittlung der Titrationskurve bei Salzzusatz wurde anstelle des Wassers den Proben eine gleiche Menge Salzlösung zugefügt, im übrigen wurde wie oben beschrieben vorgegangen. Da die Probenabmessungen sorgfältig eingehalten und die Proben der selben Serie aus den gleichen Jahrringen ausgeschnitten wurden, stimmte das Verhältnis Lösungsvolumen zu Holzgewicht für eine Titrationskurve gut überein.

Die Abbildungen I, 2, 3 und 4 stellen die Titrationskurven des Tannenholzes in Elektrolyten $\left(0, \mathrm{I} \mathrm{N} \mathrm{K}_{2} \mathrm{SO}_{4}\right.$, $0,5 \mathrm{~N} \mathrm{~K}_{2} \mathrm{SO}_{4}$ und $\mathrm{I}, \mathrm{ON} \mathrm{K} \mathrm{NO}_{4}$ ) und Wasser (durchgezogene Kurven) und deren Differentialkurven (gestrichelte Kurve) dar. Das Maximum der Differentialkurve entspricht dem Wendepunkt der Titrationskurve. Die Gestalt der Titrationskurven deutet darauf hin, $\mathrm{da} ß$ es sich beim Holz erwartungsgemäß um eine schwache Polysäure handelt. Die aktiven Gruppen des Holzes werden erst während der Titration dissoziiert, was sich in einem Anstieg des $\mathrm{pH}$-Wertes bereits in der Anfangsphase der Titration widerspiegelt. Mit

\section{IN WASSER}

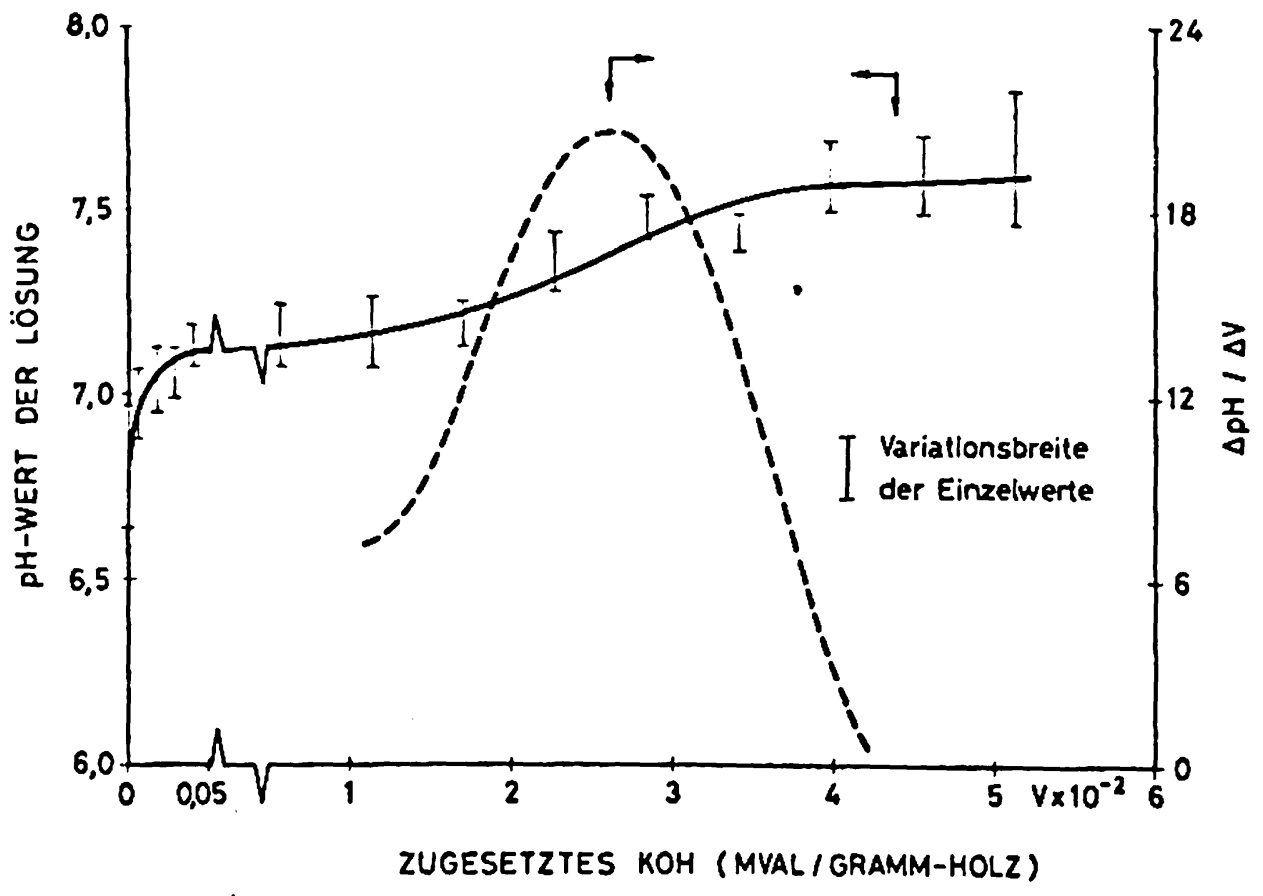

Abb. I. pH-Titrationskurve von Tannenholz in Wasser und deren Differentialkurve. Die Kurve entstand aus insgesamt 44 Einzelmessungen. Das Verhältnis Lösungsvolumen zu Holzgewicht beträgt $33,4 \pm 0,9 \mathrm{ml}:$ I g

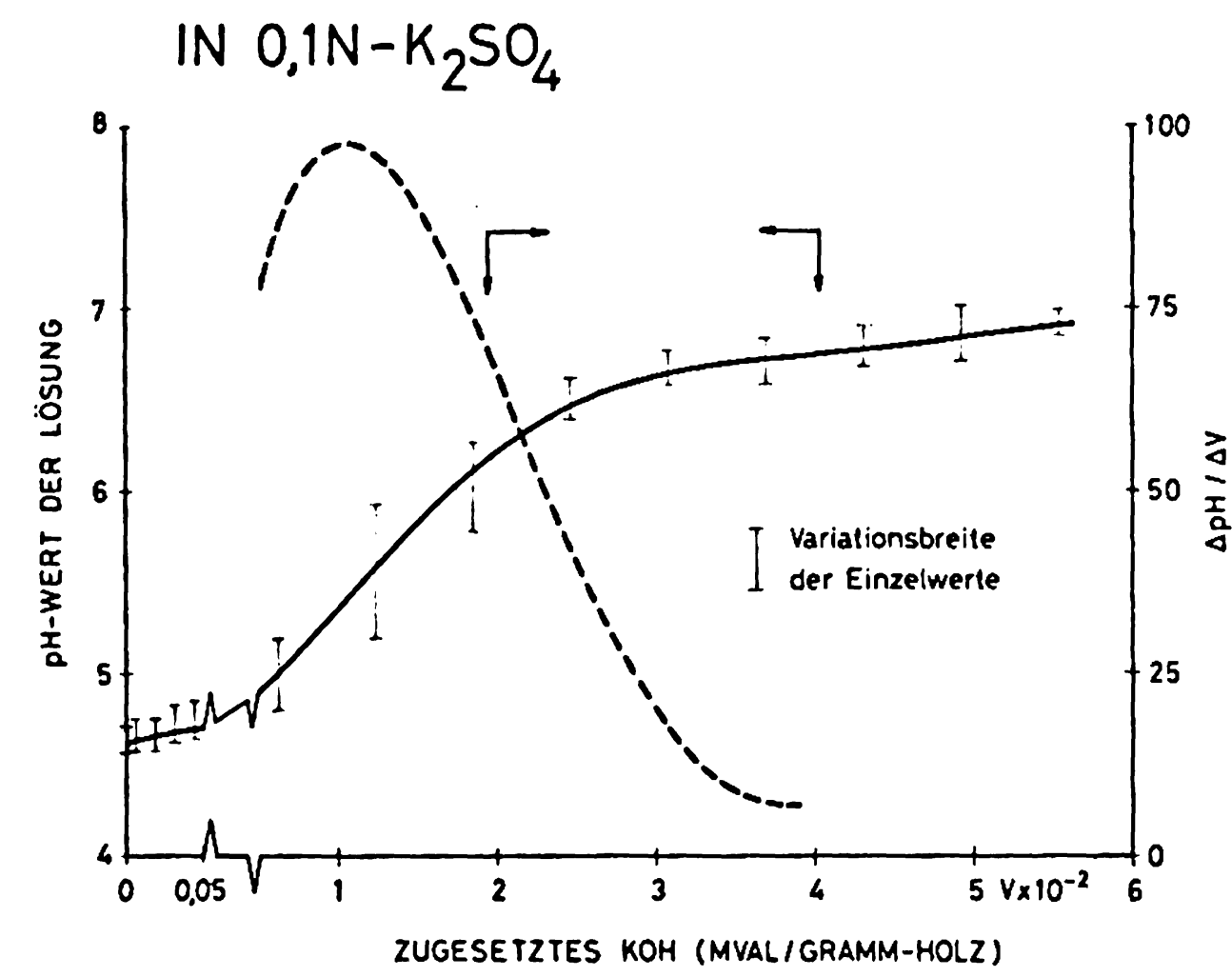

Abb. 2. pH-Titrationskurve von Tannenholz in $0, \mathrm{IN}-\mathrm{K}_{2} \mathrm{SO}_{4}$ und deren Differentialkurve. Die Kurve entstand aus insgesamt 70 Einzelmessungen. Das Verhältnis Lösungsvolumen zu Holzgewicht beträgt $28, \mathrm{I} \pm 0,6 \mathrm{ml}:$ I g

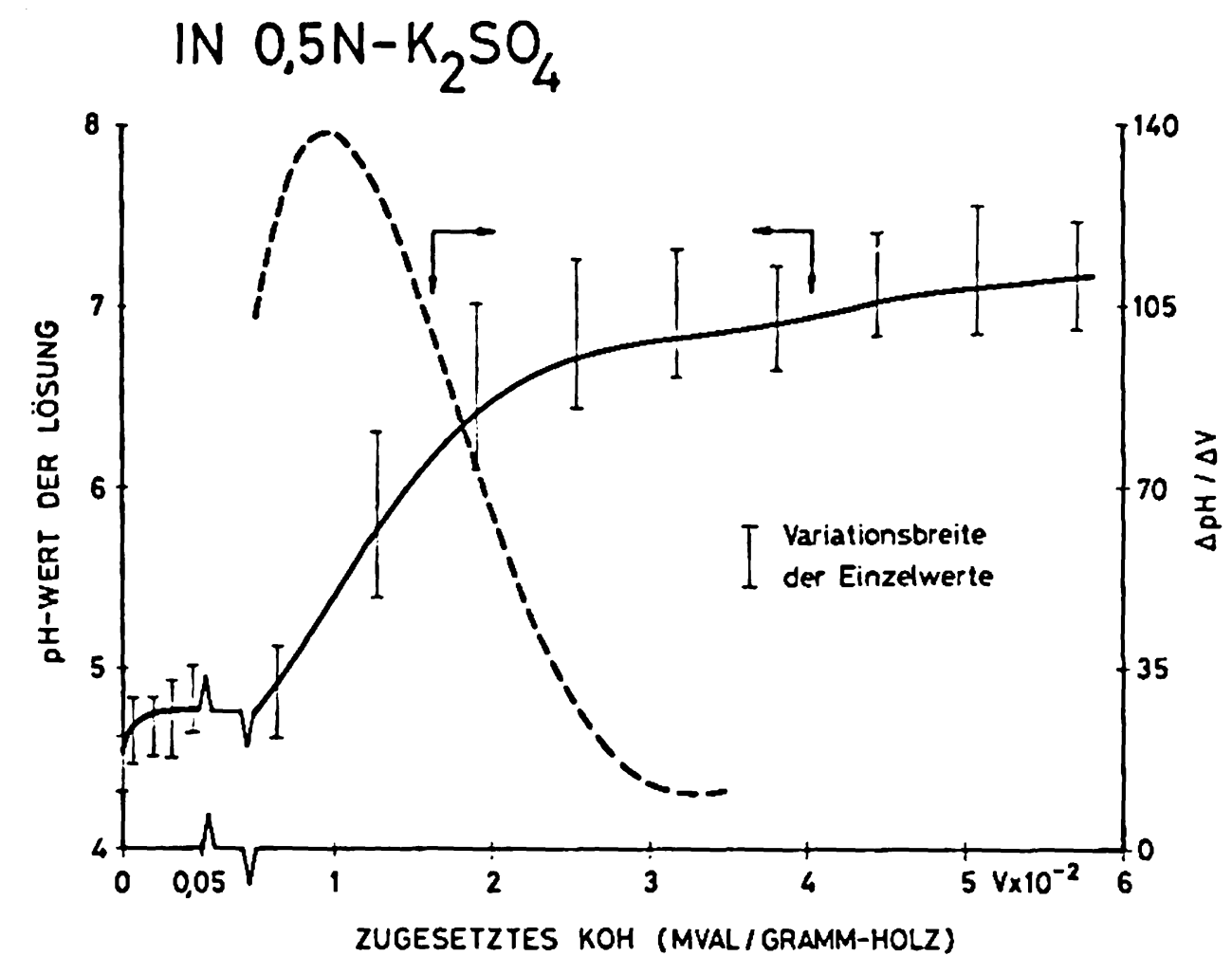

Abb. 3. $\mathrm{pH}$-Titrationskurve von Tannenholz in $0,5 \mathrm{~N}-\mathrm{K}_{2} \mathrm{SO}_{4}$ und deren Differentialkurve. Die Kurve entstand aus insgesamt 8o Einzelmessungen. Das Verhältnis Lösungsvolumen zu Holzgewicht beträgt 29,0 $\pm 0,7 \mathrm{ml}:$ I g

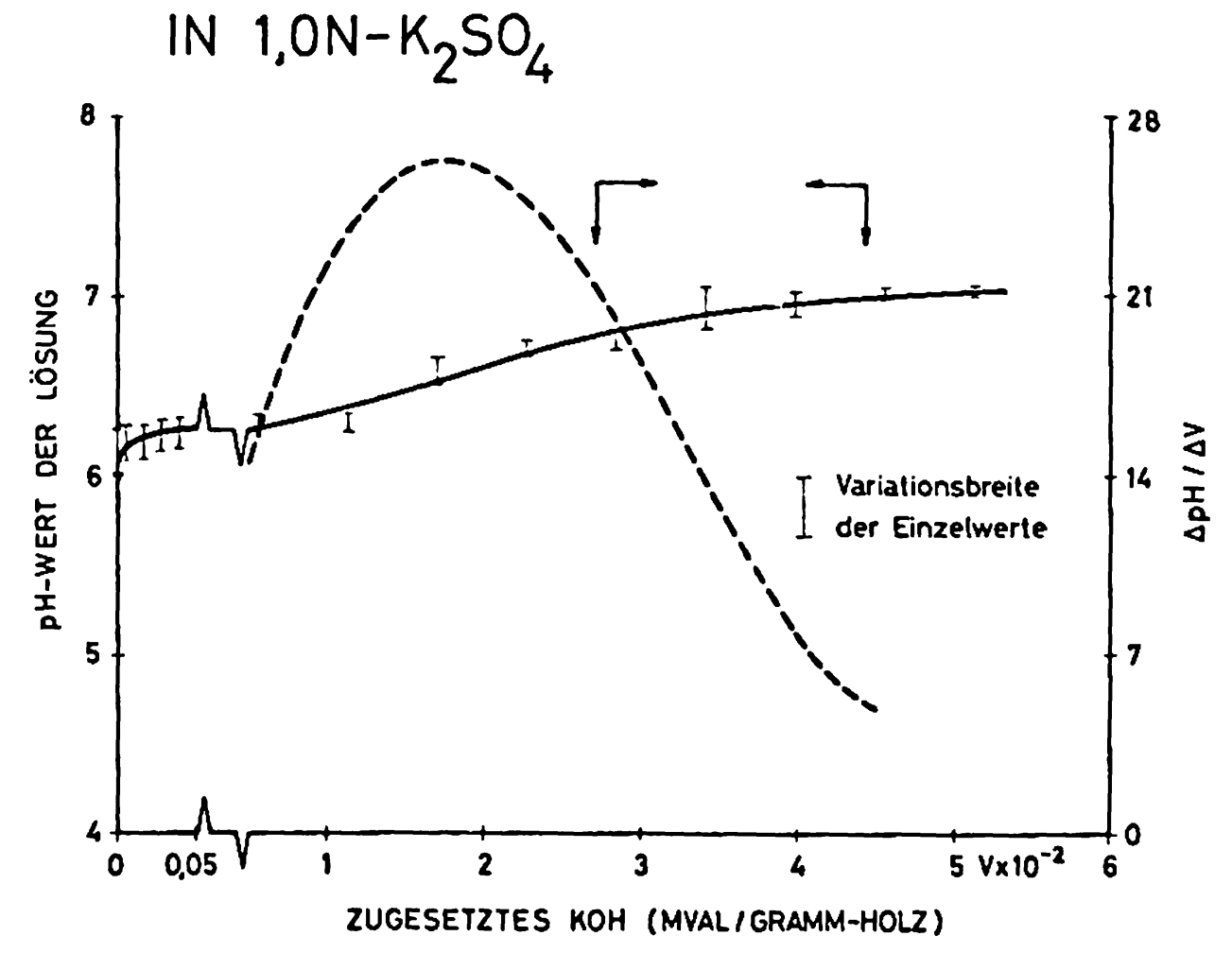

Abb. 4. pH-Titrationskurve von Tannenholz in $\mathrm{I}, \mathrm{ON}-\mathrm{K}_{2} \mathrm{SO}_{4}$ und deren Differentialkurve. Die Kurve entstand aus insgesamt 54 Einzelmessungen. Das Verhältnis Lösungsvolumen zu Holzgewicht beträgt $33,4 \pm \mathrm{I}, \mathrm{I} \mathrm{ml}:$ I $\mathrm{g}$ 
Tabelle I

Stoffbilanzentlang der Protolyse von Tannenholz mit und ohne Salzzusatz

\begin{tabular}{|c|c|c|c|c|c|c|c|c|}
\hline \multirow{2}{*}{$\begin{array}{c}\text { Zugefügtes } \\
\text { KOH } \\
\text { (mval) }\end{array}$} & \multicolumn{4}{|c|}{$\begin{array}{c}\text { Gewichtsänderung während der Protolyse } \\
\left(\begin{array}{c}\% \\
\%\end{array}\right)\end{array}$} & \multicolumn{4}{|c|}{$\begin{array}{l}\text { Beladung mit } \mathrm{K}+\text {-Ionen } \\
\text { (mval/g-Holz) }\end{array}$} \\
\hline & $\mathrm{H}_{2} \mathrm{O}$ & $\begin{array}{c}0, \mathrm{IN}- \\
\mathrm{K}_{2} \mathrm{SO}_{4}\end{array}$ & $\begin{array}{c}0,5 \mathrm{~N}- \\
\mathrm{K}_{2} \mathrm{SO}_{4} \\
\end{array}$ & $\begin{array}{c}\mathrm{I}, \mathrm{ON}- \\
\mathrm{K}_{2} \mathrm{SO}_{4}\end{array}$ & $\mathrm{H}_{2} \mathrm{O}$ & $\begin{array}{c}\mathrm{O}, \mathrm{IN}- \\
\mathrm{K}_{2} \mathrm{SO}_{4}\end{array}$ & $\begin{array}{c}0,5 \mathrm{~N}-1 \\
\mathrm{~K}_{2} \mathrm{SO}_{4}\end{array}$ & $\begin{array}{c}\mathrm{I}, \mathrm{ON}- \\
\mathrm{K}_{2} \mathrm{SO}_{4}\end{array}$ \\
\hline I & 2 & 3 & 4 & 5 & 6 & 7 & 8 & 9 \\
\hline 0 & $-0,68$ & 0,73 & 0,22 & $5,5 \mathrm{I}$ & $0,0 \mathrm{r}$ & 0,26 & 0,23 & 0,12 \\
\hline $1,948 \cdot 10^{-4}$ & $-0,44$ & $0,5 \mathrm{I}$ & 0,53 & 4,23 & 0,01 & 0,42 & 0,37 & 0,27 \\
\hline $5,843 \cdot 10^{-4}$ & $-0,49$ & 0,44 & 0,60 & 4,18 & 0,02 & $0,4 \mathrm{I}$ & 0,35 & 0,27 \\
\hline $9,739 \cdot 10^{-4}$ & $-0,78$ & 0,38 & 0,50 & 4,19 & 0,04 & $0,4 \mathrm{I}$ & 0,34 & 0,27 \\
\hline $1,363 \cdot 10^{-3}$ & $-0,45$ & $0,5 \mathrm{I}$ & 0,42 & 4,13 & 0,06 & 0,43 & 0,35 & 0,27 \\
\hline $1,948 \cdot 10^{-2}$ & $-0,63$ & 0,42 & 0,46 & 4,28 & 0,22 & 0,48 & 0,43 & 0,34 \\
\hline $3,895 \cdot 10^{-2}$ & $-0,80$ & 0,56 & 0,55 & 3,69 & 0,45 & 0,65 & 0,62 & 0,53 \\
\hline $5,843 \cdot 10^{-2}$ & $-0,43$ & 0,66 & 0,53 & 5,29 & 0,61 & 0,83 & 0,82 & 0,78 \\
\hline $7,791 \cdot 10^{-2}$ & $-0,66$ & 0,81 & 0,36 & 4,51 & 0,87 & 1,04 & 1,05 & 0,98 \\
\hline $9,739 \cdot 10^{-2}$ & $-0,79$ & 0,49 & 0,20 & 4,36 & $\mathrm{I}, 08$ & 1,22 & 1,28 & 1,15 \\
\hline $\mathrm{I}, \mathrm{I} 6 \mathrm{~g}^{\cdot} \cdot \mathrm{IO}^{-1}$ & $-0,62$ & 0,73 & 0,10 & 4,00 & $I, 26$ & $r, 49$ & 1,60 & 1,38 \\
\hline $1,363 \cdot 10^{-1}$ & $-0,73$ & 0,95 & 0,04 & 3,82 & I,48 & 1,69 & 1,72 & $\mathrm{I}, 48$ \\
\hline $1,55^{8} \cdot 10^{-1}$ & $-0,50$ & 0,69 & 0,20 & 3,87 & $I, 64$ & $r, 99$ & 1,98 & 1,87 \\
\hline $1,753 \cdot 10^{-1}$ & $-0,60$ & 0,16 & 0,35 & 3,73 & 1,84 & 2,03 & $2, I I$ & $I, 94$ \\
\hline
\end{tabular}

fortschreitender Titration werden die sauren Gruppen neutralisiert und der $\mathrm{pH}$-Wert der Lösung steigt steiler an. Infolge des steigenden $\mathrm{pH}$-Wertes dissoziieren auch die schwächer sauren Gruppen, was zu einer Abflachung der Titrationskurve führt. Wie aus diesen Titrationskurven hervorgeht, beträgt die nutzbare Kapazität des Tannenholzes etwa 0,0I $6 \pm 0,007 \mathrm{mval}$ bezogen auf das Trockengewicht. Unter diesen Versuchsbedingungen zeigt sich kein bemerkenswerter Unterschied in der in Wasser oder in Salzlösungen bestimmten Kapazität. Der Salzzusatz bewirkt lediglich eine Verschiebung der Kurvenverläufe zu den niedrigeren $\mathrm{pH}$-Werten.

Tabelle I gibt Auskunft über die Stoff bilanz entlang der Protolyse in An- und Abwesenheit von neutralem Salz. Wie aus der Tabelle ersichtlich ist, bleibt die maximale Beladung mit Gegenionen praktisch gleich, ob die Titration in An- oder Abwesenheit des Salzes erfolgt. In der unteren pH-Region der Sorptionskurve (siehe auch Abb. 5) besteht in dieser Hinsicht ein deutlicher Unterschied. Ist die Beladung mit $\mathrm{K}^{+}$-Ionen in wäßrigem Milieu unbedeutend, steigt diese beim Salzzusatz merklich an und zwar sogar dort, wo kein Titrationsmittel zugefügt worden ist (Kolonnen $6,7,8$ und 9). Dies deutet darauf hin, daß die total aufgenommene $\mathrm{K}^{+}$-Menge nur auf das vorhandene Salz zurückzuführen ist. Die Gewichtsänderungen (Kolonnen 2, 3, 4 und 5) unterscheiden sich hingegen merklich voneinander. Bewirkt das Titrationsmittel allein eine Gewichtsabnahme, so ist beim Salzzusatz eine Gewichtszunahme zu verzeichnen, die die Beladung mit Gegenionen weit überschreitet. Die Gewichtsänderung stellt nur ein bedingt geeignetes $M a ß$ für den Sorptionsgrad dar, da hiermit nicht die qualitativen Belange erfaßt werden, sie liefert aber trotzdem einige Hinweise auf die Sorptionsart. Die Gewichtsänderungen während der Ionenaufnahme deutet darauf hin, daß der Ionenaustausch nicht der einzige Sorptionsmechanismus darstellt. Das Holz tauscht nicht nur Gegenionen aus, sondern nimmt auch, je nach Konzentration, mehr oder weniger zu den Gegenionen gehörende, doch entgegengesetzt geladene Ionen auf. Damit aber die Neutralität von Holz und Lösung aufrecht erhalten bleibt, folgt den Begleitionen eine äquivalente Menge von Gegenionen in das Holz. Diese Ionenverteilung zwischen den zwei Phasen kann mit der Donnan'schen Theorie erklärt werden. Der Rest der Gewichtszunahme ist auf das Salz zurückzuführen, welches durch die van der Waals'schen- und Kapillarkräfte gebunden ist.

\subsection{Bestimmung von Dissoziationskonstanten}

Die scheinbare Dissoziationskonstante des Holzes ist durch den $\mathrm{pK}$-Wert der ionenaktiven Molekülgruppen der Holzsubstanz gegeben.

In der Literatur sind verschiedene Methoden beschrieben, mit denen sich die Dissoziationskonstante je nach Genauigkeitsanforderungen bestimmen läßt. Grundsätzlich können diese Methoden in zwei Gruppen eingeteilt werden. Die erste führt über recht aufwendige Verfahren zur Bestimmung von Ionenaktivitäten und somit zu thermodynamischen Dissoziationskonstanten, die zweite über Titrationskurven zu konzentrationsabhängigen Gleichgewichtskonstanten. Wir haben uns bei der Bestimmung der Aciditätskonstanten von Holz Methoden der zweiten Gruppe bedient. Die üblichste Methode beruht auf der Gleichung 5 (Griessbach 1957) oder Gleichung 7 (Helfferich 1959), in der die Gleichgewichtskonstante dem pHWert im Halbneutralisationspunkt entspricht. Andere Verfahren benutzen die Größe des Potentialsprungs in der Nähe des Schnittpunktes (Jenkins und Latham I966) oder arbeiten nach der Bestimmung der Pufferkapazität (Gregor und Bergman I948). Es soll hier bemerkt werden, daß die genaue Berechnung der Dissoziationskonstanten von Polysäuren, sogar der monofunktionellen, mit Schwierigkeiten verbunden ist. Diese Schwierigkeiten bestehen darin, daß bei der fortschreitenden Neutralisation die Dissoziationskonstante mit dem Dissoziationsgrad variiert. Die bereits ionisierten Gruppen bilden ein elektrisches Feld, das anschließend zur weiteren Protonen-Abgabe des Polyelektrolyten führt (Gregor, Luttinger und Loebel 1959). Beim polyfunktionellen Holz treten zu den bereits erwähnten Komplikationen noch weitere hinzu. Bei der Beladung des Polyelektrolyten gehören zu jedem Gleichgewichts- 
pH-Wert verschiedene Dissoziationskonstanten, deren Werte den einzelnen funktionellen Gruppen entsprechen. Es erübrigt sich in diesem Fall eine Durchschnittskonstante zu berechnen, die der Hälfte der totalen Austauschkapazität entsprechen würde. Vielmehr ist eine approximative Berechnung sinnvoll, die die einzelnen Abschnitte der Titrationskurve in Betracht zieht und für jeden Kurven-Abschnitt die entsprechende Gleichgewichtskonstante bestimmt. Die Unsicherheit dieser Methode liegt darin, daß die Titrationskurve meistens keine deutliche Inflexionspunkte aufweist. Gemäß Gleichung 7 ist für die Ermittlung der scheinbaren Dissoziationskonstanten von $\mathrm{Holz}$ außer der Kenntnis des pH-Wertes der Lösung und der Konzentration der aktiven Gruppen auch noch der Beladungszustand notwendig. Den pH-Wert der Lösung kann man aus der Titrationskurve für die Bestimmung der Sorptionskapazität entnehmen. Die Konzentration der aktiven Gruppen wird aus der zugesetzten Basen- bzw. Salz-Menge berechnet. Für die Ermittlung der Gegenionen-Konzentration ist jedoch eine gesonderte experimentelle Bestimmung erforderlich.

Die analytische Methode für die Bestimmung der Gegenionen-Konzentration beruht auf der Annahme, $\mathrm{daB}$ die durch das $\mathrm{Holz}$ aufgenommene KationenMenge derjenigen der freigewordenen Wasserstoffionen entspricht (Topp und Pepper 1949). Man bedient sich dabei der Proben für die Bestimmung der nutzbaren Ionen-Sorptionskapazität, indem man aus den einzelnen Schliffkolben eine bestimmte Menge Lösung pipettiert und mit eingestellter $0,02 \mathrm{~N} \mathrm{KOH}$ oder $0,02 \mathrm{~N}$ $\mathrm{H}_{2} \mathrm{SO}_{4}$ bis zu pH $=7$ titriert. Auf die gleiche Weise wurde eine Kontrollreihe gleicher Lösungen, jedoch ohne Proben titriert, um den Verbrauch der Probenreihe zu korrigieren.

Für die Schätzung der Dissoziationskonstanten von einzelnen, im Holz befindlichen ionogenen Gruppen zeigt sich die Auftragung des Beladungszustandes von Holz gegen den $\mathrm{pH}-$ Wert der Lösung sinnvoll. In Abb. 5 sind solche Kurven dargestellt, wobei mit und ohne Salzzusatz gearbeitet worden ist. Die Verschiebung der Titrationskurve in Anwesenheit eines neutralen Salzes $\left(\mathrm{K}_{2} \mathrm{SO}_{4}\right)$ deutet darauf hin, daß es beim $\mathrm{Holz}$ zu einem Ionenaustausch gekommen ist. Wie aus den Kurvenverläufen ersichtlich ist, beginnt der Ionenaustausch mit dem Zusetzen von Salz, d. h. noch bevor Titrationslösung zugefügt worden ist. Die durch den Ionenaustausch in die Lösung gelangten Wasserstoffionen erniedrigen deren $\mathrm{pH}$-Wert.

Die thermodynamische Dissoziationskonstante ist eine Konstante im wahrsten Sinne des Wortes. Sie beruht auf Ionen-Aktivitäten und charakterisiert bei einer bestimmten Temperatur das Dissoziationsgleichgewicht. Die experimentelle Bestimmung von Ionenaktivitäten stößt auf erhebliche Schwierigkeiten, besonders wenn es sich um hochmolekulare Ionen handelt. Beim Ersetzen von Aktivitäten durch Konzentration erhält man eine "scheinbare" Dissoziationskonstante $\left(\mathrm{K}_{C}\right)$, die von der Ionenstärke abhängig ist.

Wie die Stufen der experimentell ermittelten Kurven (Abb. 5) erkennen lassen, sind im Holz zwei funktionelle Gruppen ionenaktiv. Mit steigendem $\mathrm{pH}$-Wert des Mediums steigt auch die Dissoziation der schwach sauren Gruppen. Die vollständige Dissoziation der ladungstragenden Gruppen erfolgt erst, wenn der pH-

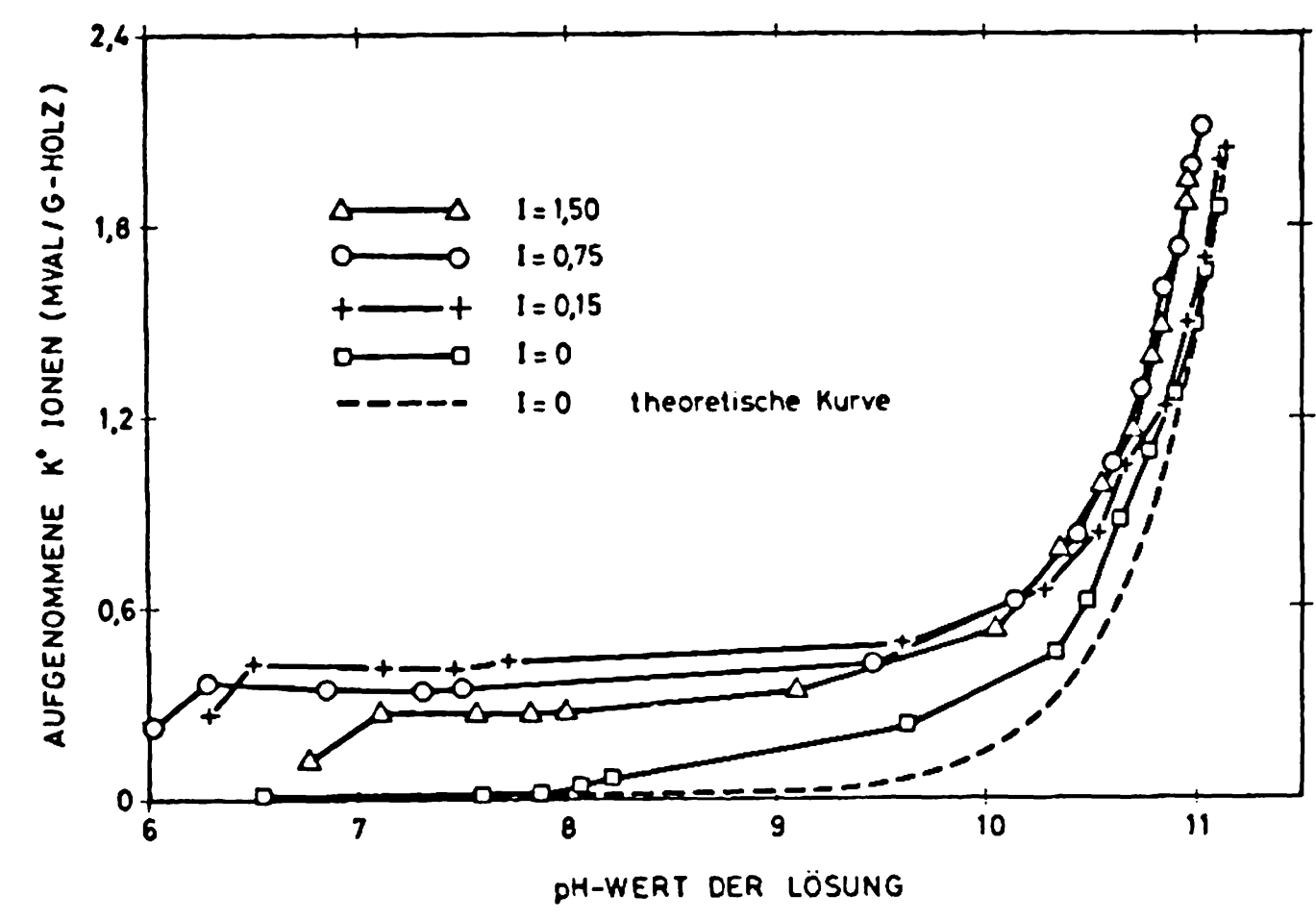

Abb. 5. Ausnutzbare Gegenionen-Sorption von Tannenholz während der Protolyse in Lösungen von verschiedener Ionenstärke $(I=0, I=0,15, I=0,75, I=I, 50)$

Wert der Lösung den pK-Wert der Gruppe erreicht. Dieser Vorgang ist reversibel, d. h. sinkt der $\mathrm{pH}$-Wert des Mediums unter den pK-Wert der Gruppe ab, so wird wieder ioneninaktives Holz zurückgebildet. Die hier bestimmten Gleichgewichtskonstanten beruhen auf analytischen Konzentrationen und sind deshalb von der Ionenstärke abhängig. Sie können als scheinbare Aciditätskonstanten angesehen werden. Der $\mathrm{pK}_{C}$-Wert der ersten Stufe beträgt $\mathrm{pK}_{C}^{\prime}=5,8 \pm 0,9$ und der zweiten Stufe $\mathrm{pK}_{c}^{\prime \prime}=10,4 \pm 0,4$. Verglichen mit den Dissoziationskonstanten der verschiedenen ionenaktiven Gruppen in Ionenaustauschern (Helfferich 1959) nähert sich die erste Stufe den Carboxyl- und die zweite Stufe den phenolischen Hydroxylgruppen. Diese Angaben können nur als hinweisende Werte angesehen werden, da, wie Hammett (1973) am Beispiel von substituierten Phenolen und Carbonsäuren gezeigt hat, die Ionisierung einer Gruppe stark von der Stellung der Elektronen an den Substituenten selbst abhängt. Solche Strukturänderungen wirken sich auf die Dissoziation der Ankergruppen aus.

Wie aus Abbildung 5 weiter zu entnehmen ist, ist das erste Maximum der Sorptionskurve von der Ionenstärke der Lösung abhängig. Eine abnehmende Tendenz des Gegenionen-Sorbatgehaltes mit steigender Ionenstärke der flüssigen Phase ist, mit Ausnahme von $\mathrm{I}=0$, deutlich. $\mathrm{Da}$ die im Wasser $(\mathrm{I}=0)$ bestimmte Gegenionen-Sorption und die nutzbare Kapazität des Tannenholzes in einem scheinbaren Widerspruch stehen, ist lediglich auf die mangelnde Ionen-Konzentration in der Titrationslösung zurückzuführen. Die hemmende Auswirkung der Ionenstärke auf die Gegenionen-Aufnahme ist mit einer stärkeren Konkurrenz um die Bindungsstellen am Holz und demzufolge mit einer geringeren Wechselwirkung zwischen dem Sorbat und den funktionellen Gruppen des Holzes zu erklären.

\section{Literatur}

Bauch, J. 1964. Die axiale Durchlässigkeit von Kiefersplintholz für wäßrige Lösungen. 2. Mitt. Elektrolyte. Planta 6r: 309-331.

Bosshard, H. H. 1977. Private Mitteilung.

Gregor, H. P. und J. I. Bergman. 1948. Characterisation of ion exchange resins. I. Acidity and number of constituens cation exchange groups. J. Am. Chem. Soc. 70: 2370-2372. 
Gregor, H. P., L. B. Luttinger and E. M. Loebel. Metalpolyelektrolyte complexes. I. The polyacrylic acid-copper complex. J. Phys. Chem. 59: 34-39.

Griessbach, R. 1957. Austauschadsorption in Theorie und Praxis. Academ. Verlag, Berlin.

Hammett, L. P. 1973. Physikalische Organische Chemie. Verlag Chemie GmbH, Weinheim.

Helfferich, F. I959. Ionenaustauscher, Bd. I. Verlag Chemie $\mathrm{GmbH}$, Weinheim.

Jenkins, D. A. and J. L. Latham. 1966. Estimation of some $\mathrm{K}_{\mathrm{i}}$ and $\mathrm{K}_{\mathrm{sp}}$ by potentiometric titration. J. Chem. Educat. 43 (2): $82-84$.

Kratzl, K. und H. Stepnicka. 1955. Über Kationenaustauscher auf Holzbasis. Mitteilungen der Österreichischen Gesellschaft für Holzforschung, Bd. 7, I. Teil, Folge 4
(20. August 1955); "Internationaler Holzmarkt" 1955, Nr. 16.

Kratzl, K. und H. Stepnicka. 2. Teil, Folge 6 (Io. Dezember 1955), „Internationaler Holzmarkt" 1955, Nr. 24.

Ohlsson, A. and S. Rydin. 1975. Washing of pulps. Part 2. The sorption of $\mathrm{Na}, \mathrm{Mg}$ and $\mathrm{Ca}$ on kraft pulp. Svensk Papperstidn. 15: 549-552. . 1

Pitman, M. G. I976. Ion uptake by plant roots. In: Encyclopedia of Plant Physiology, New Series (Editors U. Lüttge, M. G. Pitman), Vol. 2. Transport in Plants II, Part B: Tissues and Organs: 122. Springer-Verlag, Berlin, Heidelberg, New York.

Rosen, A. 1975. Adsorption of sodium ions on kraft pulp fibers during washing. Tappi 58 (9): I56-I6I.

\title{
Étude de la Mouillabilité du Bois
}

\author{
Par Gérard Elbez
}

Centre Technique du Bois, Paris

Mots-clés

Adhésifs

Angle de contact ou angle de raccordement

Energie de mouillage

Energie d'adhésion

Enthalpie libre interfaciale

Qualité de collage
Keywords

Adhesives

Contact angle

Free energy of wetting

Free energy of adhesion

Surface tension

Glue bond quality
Schlüsselwörter

(Sachgebiete)

Adhäsionsenergie

Benetzbarkeit

Benetzungsenergie

Bindefestigkeit

Bindemittel

Kontaktwinkel

Oberflächenspannung

Étude de la Mouillabilité du Bois

\section{Résumé}

L'étude a porté sur le mouillage de différents supports: verre, Hêtre, Sapin et Chêne par de l'eau, afin de définir un mode opératoire. Cette méthode a été transposée ensuite aux adhésifs qui font l'objet de cette étude. Ce travail a porté sur des adhésifs courants, à savoir: urée-formol, urée-formol plastifiée, résorcine, vinylique et vinylique à durcisseur, ainsi que sur des adhésifs modifiés par incorporation de tensio-actifs.

Lors des essais de mouillage a été mesuré l'angle de contact bois - adhésifs $(\theta)$, puis les énergies de mouillage et d'adhésion ont été calculées. Parallèlement, des essais de collage ont été effectués et leurs caractéristiques mesurées: résistance au cisaillement en traction et pourcentage de rupture dans le bois (adhérence). De ces essais, il ressort que l'étude de la mouillabilité des bois par des colles de nature chimique voisine permet une sélection des formulations susceptibles d'optimaliser la qualité du collage.

\section{Study of the Wettability of Wood}

\section{Summary}

The study relates to the wetting of different solid surfaces: glass, beech, fir and oak to define a procedure. This method was applied to adhesives: several classical adhesives were considered: urea formaldehyde, urea formaldehyde plastified, vinyl acetate, vinyl acetate with hardener and adhesives modified by surface active agents added.

During the experiments, we have measured the contact angle of adhesives on wood substrate and calculated free energies of wetting and adhesion. Simultaneously the glue bond qualities were controlled and their characteristics measured in other words: bond strengh and adhesion. These experiments have established that the wettability of wood by adhesives which present few chemical differences allow to choose adhesives to obtain an optimum glue bond quality.

\section{Untersuchungen über die Benetzbarkeit des Holzes}

\section{Zusammenfassung}

Untersucht wurde die Benetzbarkeit unterschiedlicher Oberflächen: Glas, Buchen-, Eichen- und Fichtenholz mit dem Ziel, eine Meßmethode für die Verleimbarkeit bzw. Leimbindung auszuarbeiten. Dazu wurden folgende Leime ausgewählt: Harnstoff-Formaldehydharz, weiches Harnstoffharz, Resorcinharz, Vinylacetat, Vinylacetat mit Härter sowie durch oberflächenaktive Stoffe modifizierte Bindemittel.

Gemessen wurde der Kontaktwinkel zwischen Leim und Holz und die Benetzungs- und Adhäsionsenergie berechnet. Gleichzeitig wurde die Qualität der Leimbindung durch die Bindefestigkeit und Adhäsion charakterisiert. Die Untersuchungen haben bestätitg, daß mit Hilfe der Benetzbarkeitmessungen von Leimen unterschiedlicher chemischer Natur solche Leime ausgewählt werden können, mit denen eine optimale Bindung erzielt werden kann.

\section{Introduction}

Parmi les conditions nécessaires pour obtenir un bon collage de deux surfaces solides, il faut citer, avec Collett (1972), en particulier:

- le durcissement de l'adhésif,

- le bon mouillage des surfaces solides par le liquide adhésif,
- l'aptitude des matériaux à se déformer pour contenir les contraintes élastiques se produisant lors de la formation du joint.

Le rôle important de la mouillabilité des supports solides sur le processus de l'adhésion semble être confirmé par de nombreux auteurs et en particulier par Herczeg (1965) et Bryant (1968). Nous avons, de ce 